REVISTA PROYECCIONES N ${ }^{\circ}$ 11: 124-145

Julio 1986 - I.S.S.N. 0716-0917

\title{
UNA APLICACION DE LAS FUNCIONES SPLINE
}

HECTOR SOZA P.*

RESUMEN.

Dada la funcion $f$, sobre un intervalo $[a, b]$, definida en los no dos $a<t_{1}<t_{2}<\ldots<t_{n-1}<t_{n}<b$ y de la cual se sabe que es discon tinua su primera derivada en un punto $c \in[a, b]$, se analiza la existencia y unicidad de la Función spline de Interpolación que aproxima a $f$ en estos nodos y acepta esta condición. Posteriormente se determina un algoritmo de cálculo explícito para la solución encontrada y se agrega un pro grama computacional que obtiene esta solución.

1. Introducción. Teoría Clásica de Funciones Spline.

Los siguientes conceptos están desarrollados en [1].

Sean $X, Y, Z$ tres espacios de Hilbert $y$ sean $\left\langle,{ }_{x} ;\left\langle,{ }_{y} ;\left\langle,{ }_{z}\right.\right.\right.$ los productos internos respectivos $\mathrm{y}\|\|_{\mathrm{x}},\|\|_{\mathrm{y}},\|\|_{\mathrm{z}}$ las normas

* Profesor Departamento de Matemáticas, Facultad de Ciencias. Universidad de La Serena - Chile. 
inducidas por estos productos internos.

Sean $\mathrm{T}: \mathrm{X} \rightarrow \mathrm{Y}$ y $\mathrm{A}: \mathrm{X} \rightarrow \mathrm{Z}$ dos aplicaciones lineales continuas. Dado z $E$ Z fijo, llamamos:

$$
I_{z}=\{f \in X / A(f)=z\}
$$

El problema a resolver es hallar $F E_{z^{\prime}}$ si existe, tal que:

$$
\left.|| \mathrm{T}(\mathrm{F})\right|_{\mathrm{Y}}=\underset{\mathrm{f}_{\varepsilon} \mathrm{I}_{\mathrm{z}}}{\min }|| \mathrm{T}(\mathrm{f}) \|_{\mathrm{Y}}
$$

F se denomina Función Spline de Interpolación (relativá a T, A y z).

\subsection{Existencia y unicidad.}

Teorema 1.1 .

Si $\operatorname{Ker}(\mathrm{T})+\operatorname{Ker}(\mathrm{A})$ es cerrado y si se verifica que $\operatorname{Ker}(\mathrm{T}) \cap \operatorname{Ker}$ $(A)=\{0\}$ entonces, dado $z \varepsilon z$, existe una única Función Spline de Interpolación (relativa a T, A Y z).

\section{Corolario 1.1.}

Si Ker(T) O Ker(A) son de dimensión finita (o de corimensión fi nita) entonces, para cualquier $z \varepsilon z$, existe una Función Spline de Interpolación (relativa a T, A y z).

\subsection{Caracterización}

Teorema 1.2

Una condición necesaria y suficiente para que $F$ E $I_{z}$ sea la Fun ción Spline de Interpolación (relativa a $T, A$ y ) es que se tenga: 


$$
\langle\mathrm{T}(\mathrm{F}), \mathrm{T}(\mathrm{f})\rangle_{\mathrm{Y}}=0, \forall \mathrm{f} \varepsilon \operatorname{Ker}(\mathrm{A})
$$

\section{Corolario 1.2}

Una condición necesaria y suficiente para que $F \varepsilon I_{z}$ sea la Función Spline de Interpolación (relativa a $T, A$ y $z$ ) es que exista $\lambda \varepsilon z$ tal que :

$$
T^{\prime}(T(F))=A^{\prime}(\lambda)
$$

o sea:

$$
T^{\prime}(T(F)) \varepsilon(\operatorname{Ker}(A)) \underline{1}
$$

donde $T^{\prime}$ y A' representan los operadores adjuntos de T y A respectivamente.

\section{Definición.}

$$
\text { Sea } S=\left\{f \varepsilon X /\langle T(f), T(g)\rangle_{y}=0, \forall g \varepsilon \operatorname{Ker}(A)\right\} . \quad S \text { se denomina }
$$

Espacio de Funciones Spline.

\subsection{Número finito de condiciones de interpolación.}

Sea $z=R^{n} y \operatorname{dim} \operatorname{Ker}(T)=q$ finita. Entonces se verifica que:

1) $\sin \geqslant q$ entonces $\operatorname{Ker}(T) \cap \operatorname{Ker}(A)=\{0\}$.

2) si se definen $k_{i}: x \rightarrow[R, i=1, \ldots, n$, funcionales lineales continuas sobre $x$, linealmente independientes, entonces:

$$
\forall f x, A(f)=\left(\begin{array}{c}
\left.<K_{1}, f\right\rangle_{x} \\
\cdot \\
\left.<K_{n}, f\right\rangle_{x}
\end{array}\right)
$$

3) $I_{z}=\left\{\mathrm{f} \varepsilon \mathrm{x} /\left\langle\mathrm{K}_{\mathrm{i}}, \mathrm{f}\right\rangle_{\mathrm{x}}=z_{i}, i=1, \ldots, \mathrm{n}\right\}$ 
$\forall z \in R^{n}, A^{\prime}(z)=\sum_{i=1}^{n} z_{i} K_{i}, A^{\prime}$ adjunta de $A$.

4) la condición expresada en el corolario 1.2 es que existan los coeficientes $\lambda_{i}, i=1, \ldots, n$, tales que:

$$
\mathrm{T}^{\prime}(\mathrm{T}(\mathrm{F}))=\sum_{i=1}^{\mathrm{n}} \lambda_{i} \mathrm{~K}_{i} \quad \mathrm{y} \quad \mathrm{T}^{\prime}(\mathrm{T}(\mathrm{F})) \varepsilon(\operatorname{Ker}(\mathrm{A}))^{\underline{1}}
$$

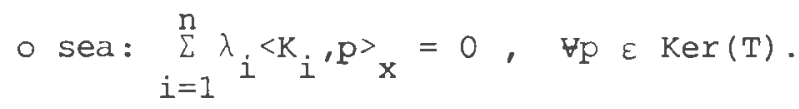

1.4. Caso particular.

$$
\begin{aligned}
& \text { Sean: } X=H^{2}[a, b]=\left[f / f, f^{\prime} y f^{\prime \prime} \text { son continuas } y\right. \text { están en } \\
& \left.L^{2}[a, b]\right\} \\
& Y=L^{2}[a, b]=\{f / f \text { es de cuadrado integrable }\} \\
& \mathrm{z}=\mathrm{R}^{\mathrm{n}} \\
& T: X \rightarrow Y \text { tal que } \forall f \in X, T(f)=\frac{d^{2} f}{d t^{2}} \\
& t_{1}, \ldots, t_{n} \text { en }[a, b] \text { tal que } a<t_{1}<t_{2}<\ldots<t_{n-1}<t_{n}<b \\
& K_{i}: x \rightarrow \mathbb{R} \text { tal que }\left\langle K_{i}, f\right\rangle_{x}=f\left(t_{i}\right), \forall f \hat{c} x, i=1, \ldots, n \\
& A: X \rightarrow z \text { tal que: } \\
& \forall f \in X, A(f)=\left(\begin{array}{c}
f\left(t_{1}\right) \\
\cdot \\
\left.\dot{t_{n}}\right)
\end{array}\right) \\
& I_{z}=\{f \varepsilon X / A(f)=z\}=\left\{f \varepsilon X / f\left(t_{i}\right)=z_{i} ; i=1, \ldots, n\right\} \\
& \text { En este caso } F \in I_{z} \text { tal que: }
\end{aligned}
$$




$$
\left.i_{a}^{b}\left(F^{\prime \prime}(t)\right)^{2} d t=\min _{f \in I_{Z}}^{b} a(f "(t))^{2} d t\right]
$$

Se verifican las condiciones del Corolario 1.1 pues:

$\operatorname{Ker}(T)=\left\{f \varepsilon X / f^{\prime \prime}(t)=0, \forall t \varepsilon[a, b]\right\}=\operatorname{polinomios}$ de grado $\{1\}$

$\operatorname{Ker}(A)=\left\{\in \in X / f\left(t_{i}\right)=0, i=1, \ldots, n\right.$ :

$\operatorname{dim} \operatorname{Ker}(\mathrm{T})=2$ y la codimensión de $\operatorname{Ker}(\mathrm{A})$ es $\mathrm{n}$. Luego, si $\mathrm{n} \geqslant 2$, se veri rican las condiciones para que exista unı Función spline, llamada spline Cúbica Natural y que se define por:

a) $F$ es polinomio de grado 1 en $\left[a, t_{1}\right]$ y $\left[t_{n}, b\right]$.

b) $F$ es polinomio de grado 3 en $\left[t_{i}, t_{i+1}\right], i=1, \ldots, n-1$.

c) F y sus derivadas hasta orden 2 son continuas.

d) $F_{n}\left(t_{i}\right)=z_{i}, i=1, \ldots, n$ (condiciones de interpolación).

e) $\underset{i=1}{n} \lambda_{i}{ }_{i}^{k}=0, k=0,1 \quad$ (condiciones de caracterización vistas en 4 ) de

\section{Función con discontinuidad en la lera. derivada.}

\subsection{Problema a estudiar.}

Dado un intervalo $[a, b]$ de $k y$ ias $t$ abscisas $t_{1}, \ldots, t_{n}$ tal que $a<t_{1}<\ldots t_{n}<b$, sea $f<c[a, b]$ tal que su primerá derivada sea discontinua en $t=c, t_{p}<c<t_{p+1}$. Dado $z=R^{n}$, el conjunto de las con diciones de interpolación para $f$ es:

$$
\left.I_{z}=\left\{f=H^{2}([a, b]-c\}\right) \cap c \mid a, b\right] ; f\left(t_{i}\right)=z_{i}, i=1, \ldots, n ;
$$

$y$ el problema es determinar $F \in I_{z}$ tal que: 
$\int_{a}^{c}\left(F^{\prime \prime}(t)\right)^{2} d t+f_{c}^{b}\left(F^{\prime \prime}(t)\right)^{2} d t=\operatorname{Min}_{f \in I}\left[\int_{a}^{c}\left(f^{\prime \prime}(t)\right)^{2} d t+i_{c}^{b}(f "(t))^{2} d t\right]$

Debido a que $\mathrm{H}^{2}([a, b]-\{c\}) \cap c[a, b]$ no es un espacio cerridu (luego no es Hilbert) y no se puede aplicar directamente la teoría clás cca expuesta. Se define un problema equivalente a (I) que sí podrá ser resugl to.

Sea $V=H^{2}[a, c] \times H^{2}[c, b]=\left\{(f, g) / f \in H^{2}[a, c]\right.$ y $g \in H^{2}[c, b]$

Se define entonces un nuevo conjunto de condiciones de interpolāción Exid $z \in \mathbb{R}^{\mathrm{n}}$ dado:

$J_{z}=\left\{(f, g) \in V / f\left(t_{i}\right)=z_{i}, i=1, ., p ; g\left(t_{j}\right)=z_{j}, j=p+1, \ldots, n ; f(c)=g(c)\right\}$

$y$ el nuevo problema es determinar $\left(F_{1}, F_{2}\right) \varepsilon J_{z}$ tal que:

$\int_{a}^{c}\left(F_{1}^{\prime \prime}(t)\right)^{2} d t+\int_{c}^{b}\left(F_{2}^{\prime \prime}(t)\right)^{2} d t=\operatorname{Min}_{\left(f, g ; \varepsilon_{z} J_{z}\right.}\left[\int_{a}^{c}\left(f^{\prime \prime}(t)\right)^{2} d t+\int_{c}^{b}(g "(t))^{2} d t\right]$

\section{Propiedad.}

Los problemas (1) y (2) son equivalentes.

En efecto, basta definir $F$ como $F_{I}$ en $[a, c]$ y $F_{2}, r_{1}[c, b]$.

\subsection{Existencia y Unicidad.}

Adaptamos el Teorema visto en 1.1. Definiendo:

$X=V=H^{2}[a, c] \quad x \quad H^{2}[c, b]$

$Y=L^{2}[a, c] \times L^{2}[c, b]$

$Z=\mathbb{R}^{n+1}$

$T: X \rightarrow Y$ dada por $T(f, g)=\left(\frac{d^{2} f}{d t^{2}}, \frac{d^{2} g}{d t^{2}}\right)$ 
$A: X \rightarrow Z$ dada por:

$$
A(f, g)=\left(\begin{array}{c}
f\left(t_{1}\right) \\
\vdots \\
f\left(t_{p}\right) \\
g\left(t_{p+1}\right) \\
\vdots \\
g\left(t_{n}\right) \\
f(c)-g(c) i
\end{array}\right.
$$

$J_{z}=\left\{(f, g) \in V / A(f, g)=\left(\begin{array}{c}z_{1} \\ \vdots \\ z_{n} \\ 0\end{array}\right\},=\left\{(f, g)=v, \begin{array}{l}f\left(t_{i}\right)=z, i=1, \ldots, p \\ g\left(t_{j}\right)=z_{j}, j=p+1, \ldots, n \\ f(c)=g(c)\end{array}\right\}\right.$

$\operatorname{Ker}(T)=\left\{(f, g) \quad \varepsilon V / f^{\prime \prime}(t)=0 y g^{\prime \prime}(t)=0, \quad \forall t \in[a, b]\right\}$

$$
\operatorname{Ker}(A)=\left\{(f, g) \in V \quad \quad \quad \begin{array}{l}
f\left(t_{i}\right)=0, \quad i=1, \ldots, p \\
g\left(t_{j}\right)=0, \quad j=p+1, \ldots, n \\
f(c)=g(c)
\end{array}\right\}
$$

Se puede comprobar que $\operatorname{Ker}(\mathrm{T})+\operatorname{Ker}(A)$ es cerrado $y$ que, si $\mathrm{n}:$ 3, $\operatorname{Ker}(T) \cap \operatorname{Ker}(A)=\left(0\right.$, . Luego existe una única solución $\left(F_{1}, F_{2}\right)$ en $J_{z}$ con $n \geqslant 3$.

\subsection{Caracterización.}

Se adaptan 1.2 y 1.3. Definiendo en Y el producto interno:

$$
\left.<\left(f_{1}, f_{2}\right),\left(g_{1}, g_{2}\right)\right\rangle_{y}=\int_{a}^{c} f_{1}(t) g_{1}(t) d t+j_{c}^{b} f_{2}(t) g_{2}(t) d t
$$

$y$ los $n+l$ funcionales $l$ ineales continuos $k_{i}, i=1, \ldots, n+l$ sobre $x$, tal que:

$$
\begin{aligned}
& \left\langle K_{i},(f, g)\right\rangle_{x}=f\left(t_{i}\right), i=1, \ldots, p \\
& \left\langle K_{j},(f, g)\right\rangle_{x}=g\left(t_{j}\right), J=p+1, \ldots, n \\
& \left\langle K_{n+1},(f, g)\right\rangle_{x}=f(c)-g(c), t_{p}<c \because t_{p+1}
\end{aligned}
$$


Sea $\left(F_{1}, F_{2}\right) \in X$, solución de (2) y $\lambda \in \mathbb{R}^{n+1}$. Las condiciones dadas en Corolario 1.2 son entonces:

$$
T^{\prime}\left(T\left(F_{1}, F_{2}\right)\right)=A^{\prime}(\lambda)=\sum_{i=1}^{n+1} \lambda_{i} K_{i}
$$

luego:

$$
\left\langle T\left(F_{1}, F_{2}\right), T(f, g)\right\rangle_{Y}=\left\langle T^{\prime}\left(T\left(F_{1}, F_{2}\right)\right),(f, g)\right\rangle_{X}=\left\langle\sum_{i=1}^{n+1} \lambda_{i} K_{i},(f, g)\right\rangle_{x}
$$

y de aquí:

$\int_{a}^{c}\left(F_{1}^{\prime \prime}(t)\right)\left(f^{\prime \prime}(t)\right) d t+\int_{C}^{b}\left(F_{2}^{\prime \prime}(t)\right)\left(g^{\prime \prime}(t)\right) d t=\sum_{i=1}^{p} \lambda_{i} f\left(t_{i}\right)+\sum_{j=p+1}^{n} \lambda_{j} g\left(t_{j}\right)+\lambda_{n+1}(f(c)-g(c))$

Por otro lado: $\sum_{i=1}^{\mathrm{n}+1} \lambda_{i} \mathrm{~K}_{i} \in \quad[\operatorname{Ker}(\mathrm{A})] \underline{\underline{1}}$, luego:

$$
\begin{aligned}
& \sum_{i=1}^{p} \lambda_{i} t_{i}^{1}+\lambda_{n+1} c^{1}=0,1=0,1 \\
& \sum_{j=p+1}^{n} \lambda_{j} t_{j}^{1}-\lambda_{n+1} c^{1}=0,1=0,1
\end{aligned}
$$

Desarrollando $f$ y $g$ según Taylor, en torno a $t=c$, con el resto en su forma integral:

$$
\begin{aligned}
& f(t)=f(c)+(t-c) f^{\prime}(c)+\int_{c}^{a}(z-t)+f^{\prime \prime}(z) d z \\
& g(t)=g(c)+(t-c) g^{\prime}(c)+\int_{C}^{b}(t-z){ }_{+} g^{\prime \prime}(z) d z
\end{aligned}
$$

donde:

$$
(t-z)_{+}=\left\{\begin{array}{cl}
t-z & \text { si } t \geqslant z \\
0 & \text { si } t<z
\end{array}\right.
$$

luego:

$$
\sum_{i=1}^{p} \lambda_{i} f\left(t_{i}\right)+\lambda_{n+1} f(c)=\sum_{i=1}^{p} \int_{c}^{a} \lambda_{i}\left(z-t_{i}\right)+f^{\prime \prime}(z) d z=
$$




$$
\begin{aligned}
& =\int_{c}^{a}\left[\sum_{i=1}^{p} \lambda_{i}\left(z-t_{i}\right)_{+}\right] f^{\prime \prime}(z) d z \\
\sum_{j=p+1}^{n} \lambda_{j} g\left(t_{j}\right)-\lambda_{n+1} g(c) & =\int_{c}^{b}\left[\sum_{j=p+1}^{n} \lambda_{j}\left(t_{j}-z\right)+g^{\prime \prime}(z) d z\right.
\end{aligned}
$$

Y sumando estas ecuaciones miembro a miembro la expresión (3) queda:

$$
\begin{aligned}
& \int_{a}^{c}\left(F_{1}^{\prime \prime}(z)\right)\left(f^{\prime \prime}(z)\right) d z+\int_{C}^{b}\left(F_{2}^{\prime \prime}(z)\right)\left(g^{\prime \prime}(z) d z=\right. \\
& \quad=\int_{a}^{c}\left[-\sum_{i=1}^{p} \lambda_{i}\left(z-t_{i}\right)_{+}\right] f^{\prime \prime}(z) d z+\int_{C}^{b}\left[\sum_{j=p+1}^{n} \lambda_{j}\left(t_{j}-z\right)_{+}\right] g "(z) d z
\end{aligned}
$$

y luego:

$$
\begin{aligned}
& F_{I}^{\prime \prime}(z)=-\sum_{i=1}^{p} \lambda_{i}\left(z-t_{i}\right)+ \\
& F_{2}^{\prime \prime}(z)=\sum_{j=p+1}^{n} \wedge_{j}\left(t_{j}-z\right)+
\end{aligned}
$$

Evaluando en $z=c y$ usando (4) se deduce que:

$$
F_{1}^{\prime \prime}(c)=E_{2}^{\prime \prime}(c)=0
$$

Luego se concluye que $F_{1}, F_{2}, F_{1}^{\prime \prime} y{ }_{2}^{\prime \prime}$ son continuas en $t=c$, pero $F_{1}^{\prime} y$ $\mathrm{F}_{2}^{\prime}$ no 10 son. Además sus terceras derivadas son continuas también en es te punto.

La caracterización de està sclución es la siguiente:

i) $F_{1}$ es polinomio de grado 1 en $\left[a, t_{1}\right]$.

ii) $F_{1}$ es polinomio de grado 3 en $\left.i t_{i}, t_{1+1}\right], i=1, \ldots, p-1, y$ en $\left[t_{p}, c\right]$.

iii) $F_{2}$ es polinomio de grado 3 en $\left[c, t_{p+1}\right]$ y $\left[t_{j}, t_{j+1}\right], j=p+1, \ldots, n-1$.

iv) $F_{2}$ es polinomio de grado 1 en $\left[t_{n}, b\right)$.

v) $\quad F_{1} y F_{2}$ son continuas en $[a, b)$. 
vi) $F_{1}^{\prime \prime}(c)=F_{2}^{\prime \prime}(c)=0$.

vii) $F_{1}^{\prime \prime \prime}$ y $F_{2}^{\prime \prime \prime}$ son continuas en $[a, b]$.

\subsection{Generalización.}

Si se conoce que la función f posee una derivada discontinua en un conjunto finito de puntos $\left\{c_{1}, \ldots, c_{m}\right\}$ de $[a, b]$ se adapta directamente el resultado anterior, obteniéndose $m+1$ funciones spline cubicas definidas en los intervalos correspondientes y que verifican las condiciones dadas anteriormente.

\section{Algoritmos de Cálculo.}

Se describen dos algoritmos que permiten determinar la solución al problema estudiado. Según la utilidad y facilidad de programación se elige uno de ellos y se agrega el programa correspondiente y una aplicución.

\subsection{Método de Transporte de Relaciones.}

Se describirá este método presentado en [2], que permite calcular los coeficientes del polinomio $F_{1}\left(F_{2}\right)$ en el intervalo $\left(t_{i}, t_{i+1}\right)$, $i=1, \ldots, p-1 \quad(i=p, \ldots, n-1)$, intercalando el paso a través de $t=c\left(t_{p}\right.$ ' $c$. $\left.t_{p+1}\right)$

Sean:

$$
V_{1}(t)=\left(\begin{array}{c}
F_{1}(t) \\
F_{1}^{\prime}(t) \\
F_{1}^{\prime \prime}(t) \\
F_{1}^{\prime \prime \prime}(t)
\end{array}\right) \quad y \quad V_{2}(t)=\begin{aligned}
& F_{2}(t) \\
& F_{2}^{\prime}(t) \\
& F_{2}^{\prime \prime}(t) \\
& F_{2}^{\prime \prime \prime}(t) \\
& V_{2}
\end{aligned}
$$

si $V_{1}\left(t_{i}^{+}\right)$es conocido entonces $F_{1}$ está determinada en $\left(t_{i},{ }_{i+1}\right)$ (mediante desarrollo de Taylor). Para ello se tiene:

a) Si se conocen dos relaciones para $v_{1}(t)$ en $t_{i}^{-}$, independientes entre si y además independientes de la condición de interpolación: 


$$
F_{1}\left(t_{i}\right)=z_{i}, \quad i=1, \ldots, p
$$

entonces es posible determinar dos relaciones linealmente independientes para $v_{1}$ en $t_{i}^{+}$, usando la continuidad de $F_{1} y$ sus derivadas hasta orden dos:

$$
F_{1}^{(k)}\left(t_{i}^{-}\right)=F_{1}^{(k)}\left(t_{i}^{+}\right), k=0,1,2
$$

La incógnita $F_{1}^{\prime \prime \prime}\left(t_{i}^{-}\right)$se reemplaza por $F_{1}\left(t_{i}^{+}\right)=z_{i} y$ así se obtiene el sistema de ecuaciones:

$$
m\left(t_{i}^{+}\right) v_{1}\left(t_{i}^{+}\right)=u\left(t_{i}^{+}\right)
$$

a partir de: $m\left(t_{i}^{-}\right) v_{1}\left(t_{i}^{-}\right)=u\left(t_{i}^{-}\right)$,

donde $\mathrm{m}$ es matriz de 2 × 4 y u $\varepsilon \mathbb{R}^{2}$.

b) A partir de las dos relaciones en $t_{i}^{+}$pueden lograrse dos en $\bar{t}_{i+1}^{-}$. Usan do el desarrollo de Taylor:

$F_{1}(t)=F_{1}\left(t_{i}^{+}\right)+\left(t-t_{i}^{+}\right) F_{1}^{\prime}\left(t_{i}^{+}\right)+\frac{\left(t-t_{i}^{+}\right)^{2}}{2} F_{1}^{\prime \prime}\left(t_{i}^{+}\right)+\frac{\left(t-t_{i}^{+}\right)^{3}}{6} F_{1}^{\prime \prime \prime}\left(t_{i}^{+}\right)$

se obtiene:

$$
\begin{aligned}
& F_{1}\left(t_{i+1}^{-}\right)=F_{1}\left(t_{i}^{+}\right)+\left(t_{i+1}^{-}-t_{i}^{+}\right) F_{1}^{\prime}\left(t_{i}^{+}\right)+\frac{\left(t_{i}^{-}-t_{i}^{+}\right)^{2}}{-} F_{i}^{\prime \prime}\left(t_{i}^{+}\right)+\frac{\left(t_{i+1}^{-}-t_{i}^{+}\right)^{3}}{6} F_{1}^{\prime \prime \prime}\left(t_{i}^{+}\right) \\
& F_{1}^{\prime}\left(t_{i+1}^{-}\right)=F_{1}^{\prime}\left(t_{i}^{+}\right)+\left(t_{i+1}^{-}-t_{i}^{+}\right) F_{1}^{\prime \prime}\left(t_{i}^{+}\right)+\frac{\left(t_{i+1}^{-}-t_{i}^{+}\right)^{2}}{2} F_{1}^{\prime \prime \prime}\left(t_{i}^{+}\right) \\
& F_{1}^{\prime \prime}\left(t_{i+1}^{-}\right)=F_{1}^{\prime \prime}\left(t_{i}^{+}\right)+\left(t_{i+1}^{-}-t_{i}^{+}\right) F_{1}^{\prime \prime \prime}\left(t_{i}^{+}\right) \\
& F_{1}^{\prime \prime \prime}\left(t_{i+1}^{-}\right)=F_{1}^{\prime \prime \prime}\left(t_{i}^{+}\right)
\end{aligned}
$$$$
y \text { de aquí: } V_{1}\left(t_{i+1}^{-}\right)=A\left(h_{i}\right) V_{1}\left(t_{i}^{+}\right), \operatorname{con} h_{i}=t_{i+1}^{-}-t_{i}^{+} y A\left(h_{i}\right) \text { matriz }
$$ 
de $4 \times 4, A\left(h_{i}\right)=\left(a_{k j}\right)$ tal que:

$$
a_{k j}= \begin{cases}1 & , k=j \\ 0 & , k>j \\ \frac{h_{i}^{j-k}}{(j-k)}, & k<j\end{cases}
$$

(1) se tiene:

Se puede verificar que: $v_{1}\left(t_{i}^{+}\right)=A\left(-h_{i}\right) v_{1}\left(t_{i+1}^{-}\right)$, luego, usando

$$
m\left(t_{i}^{+}\right) A\left(-h_{i}\right) v_{1}\left(t_{i+1}^{-}\right)=u\left(t_{i}^{+}\right)
$$

denominando: $m\left(t_{i+1}^{-}\right)=m\left(t_{i}^{+}\right) A\left(-h_{i}\right) \quad y \quad u\left(t_{i+1}^{-}\right)=u\left(t_{i}^{+}\right)$

se tiene: $m\left(t_{i+1}^{-}\right) v_{1}\left(t_{i+1}^{-}\right)=u\left(t_{i+1}^{-}\right)$

- sea, dos relaciones linealmente independientes para $v_{1}(t)$ en $t=t_{i+1}{ }^{-}$. Se vuelve al paso a) para $i=1, \ldots, p$.

c) En el intervalo $\left(t_{p}, t_{p+1}\right]$. Se realiza el paso b) en $t=t_{p}$ y se ob tiene en $t=c$.

$$
m\left(c^{-}\right) v_{1}\left(c^{-}\right)=u\left(c^{-}\right)
$$

Para usar a) se aprovecha el hecho que $F_{1}(c)=F_{2}(c)$ y que $F_{1}^{\prime \prime} y F_{1}^{\prime \prime \prime}$ son continuas en el punto $c$.

La incógnita $F_{1}^{\prime}\left(c^{-}\right)$se reemplaza por: $F_{1}^{\prime \prime}\left(c^{-}\right)=F_{2}^{\prime \prime}\left(c^{+}\right)=0$. Se obtiene:

$$
m\left(c^{+}\right) v_{I}\left(c^{+}\right)=u\left(c^{+}\right)
$$

De aquí se va al paso b) usando ahora el vector $v_{2}(t)$ ya definido.

d) Sobre $\left[a, t_{1}\right], F_{1}$ es un polinomio de grado 1 , luego: 


$$
E_{1}^{\prime \prime}\left(t_{1}^{-}\right)=F_{1}^{\prime \prime \prime}\left(t_{1}\right)=0
$$

independientes entre $\mathrm{sl}_{\mathrm{y}}$ de $F_{1}\left(t_{1}\right)=z_{1}$. Aplicando la fase a) se obtienen dos relaciones para $v_{1}\left(t_{1}^{+}\right)$y luego, por fase $\left.b\right)$, dos relaciones para $v_{1}\left(t_{2}^{-}\right)$.

e) Sobre $\left(t_{n}, b\right], F_{2}$ es un polinomio de grado 1. Entonces:

$$
F_{2}^{\prime \prime}\left(t_{n}^{+}\right)=F_{2}^{\prime \prime \prime}\left(t_{n}^{+}\right)=0
$$

independientes entre si $y$ de $F_{2}\left(t_{n}\right)=z_{n}$. Se aplican fases a) y b) en sentido inverso para obtener dos ecuaciones para $v_{2}$ en $t_{n-1}^{+} y$ así suce sivamente.

Estas cinco fases permiten determinar las Funciones Spline cúbi cas de Interpolación buscadas. Aplicarto significa resolver un sistema de 4 ecuaciones y 4 incógnitas en cada intervalo $\left[t_{i}, t_{i+1}\right]$, incluido el paso por $t=c$. La construcción y resolución de estos sistemas hacen encarecer el cálculo numérico involucrado, por lo que no resulta ser el método más adecuado de utiıizar.

3.2. Cálculo explícito de las Funciones spline.

Se adapta un algoritmo desarrollade en [3].

Sean los nodos $t_{1}, \ldots, t_{n}$ tal que $a<t_{1}<t_{2}<\ldots<t_{n}<b$.

Sea $F(t)=A_{i}\left(t-t_{1}\right)^{3}+B_{I}\left(t-t_{-}\right)^{2}+C_{I}\left(t-t_{i}\right)+D_{i}$ el polinomio

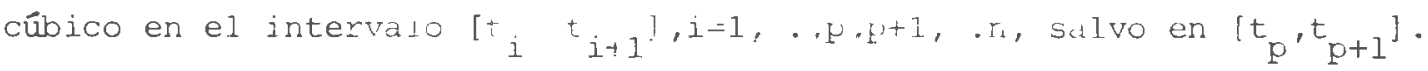
Se define:

$$
\begin{array}{ll}
n_{i}=t_{i+1} \cdot \tau_{i} & i-1, \ldots, n-1 \\
y_{i}=F\left(t_{i}\right) & i=1, \ldots, n \\
S_{i}=F^{\prime \prime}\left(t_{i}\right) & 1=1, \ldots, n
\end{array}
$$


Para determinar los coeficientes del polinomio $F(t)$, se deduce el siguiente sistema de ecuaciones lineal y tridiagonal:

$h_{i-1} S_{i-1}+2\left(h_{i-1-} h_{i}\right) s_{i}+h_{i} s_{i+1}=6\left(\frac{y_{i+1}-y_{i}}{h_{i}}-\frac{y_{i}-y_{i-1}}{h_{i-1}}\right) \quad i=2, \ldots, n-1$

donde se asume que $s_{1}=s_{n}=0$, pues en $\left[a, t_{1}\right] y\left[t_{n}, b\right] F$ es un polinomio de grado $1, s_{i}$ es la 2 da. derivada de $f$ en $t_{i}$.

Las soluciones del sistema (1), $\mathrm{S}_{2}, \ldots, \mathrm{s}_{\mathrm{n}-1}$, permiten calcular:

$$
\begin{array}{ll}
A_{i}=\frac{S_{i+1}{ }^{-S_{i}}}{6 h_{i}} & i=1, \ldots, n-1 \\
B_{i}=\frac{S_{i}}{2} & i=1, \ldots, n-1 \\
C_{i}=\frac{y_{i+1}-y_{i}}{h_{i}}-\frac{2 h_{i} S_{i}+h_{i} S_{i+1}}{6} & i=1, \ldots, n-1 \\
D_{i}=y_{i} & \\
& \\
&
\end{array}
$$

luego la Función spline cúbica es:

$$
F(t)=\left\{\begin{array}{cc}
y_{1}+C_{1}\left(t-t_{1}\right) & a \leqslant t \leqslant t_{1} \\
y_{i}+C_{i}\left(t-t_{i}\right)+B_{i}\left(t-t_{i}\right)^{2}+A_{i}\left(t-t_{i}\right)^{3} & t_{i} \leqslant t \leqslant t_{i+1} \\
\vdots & i=1, \ldots, n-1 \\
y_{n}+c_{n-1}\left(t-t_{n}\right) & t_{n} \leqslant t \leqslant b
\end{array}\right.
$$

Basta definir $F_{1}$ y $F_{2}$ en los intervalos $\left[t_{p}, c\right]$ y $\left[c, t_{p+1}\right]$ respectivamente. Se puede comprobar que:

$$
F_{1}(t)=y_{p}+C_{p}\left(t-t_{p}\right)-3 A_{p}\left(c-t_{p}\right)\left(t-t_{p}\right)^{2}+A_{p}\left(t-t_{p}\right)^{3}, \quad t p \leqslant t \leqslant c
$$




$$
F_{2}(t)=y_{p}+C_{p}\left(c-t_{p}\right)-2 A_{p}\left(c-t_{p}\right)^{3}+A_{p}(t-c)^{3}, \quad c \leqslant t \leqslant t_{p+1}
$$

es la definición para los polinomios cưbicos buscados, o sea su expresión explícita en esos intervalos.

El sistema de ecuaciones tridiagonal (1) posee una solución fácil de obtener computacionalmente y se simplifica aún más si los nodos es tán equiespaciados.

\section{Aplicación y Conclusiones.}

Se programó el método elegido, utilizando lenguaje Basic, en un computador IBM Sistema 36 perteneciente a la Universidad de La Serena. Este programa determina fundamentalmente los coeficientes de la función Spline buscada. resolviendo para ello el sistema tridiagonal asociado.

Se aplicó a la función:

$$
F(x)=x^{2 / 3}, \quad x \in(-1,1,1.1)
$$

cuya derivada posee una discontinuidad en $c=0$. Anexo a este trabajo se encuentran el programa y los resultados obtenidos en el ejemplo dado.

Se pudo comprobar que la Spline así obtenida resulta ser una aproximación muy aceptable a la función dada y mantiene la condición de discontinuidad de su primera derivada en $c=0$. 
NOMEFE: FROGFAMA ALTUAA. FIJNSFI.

NOMAEF ULTIMA HJW. UTILIZARA -... L.FFOSA79

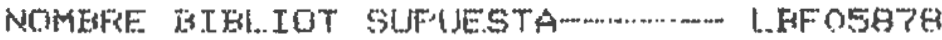

FINSSFl.. +L.GFO5B78

$05 / 08 / 85 \quad 14: 5 \%: 49$

NIMEFO RFFELENCIA MOLIFTCACION

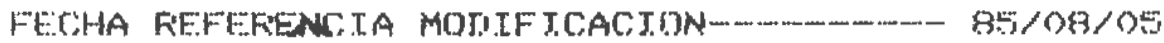

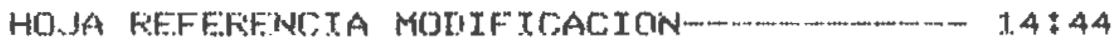

$$
\text { FUF N TE A S T C }
$$

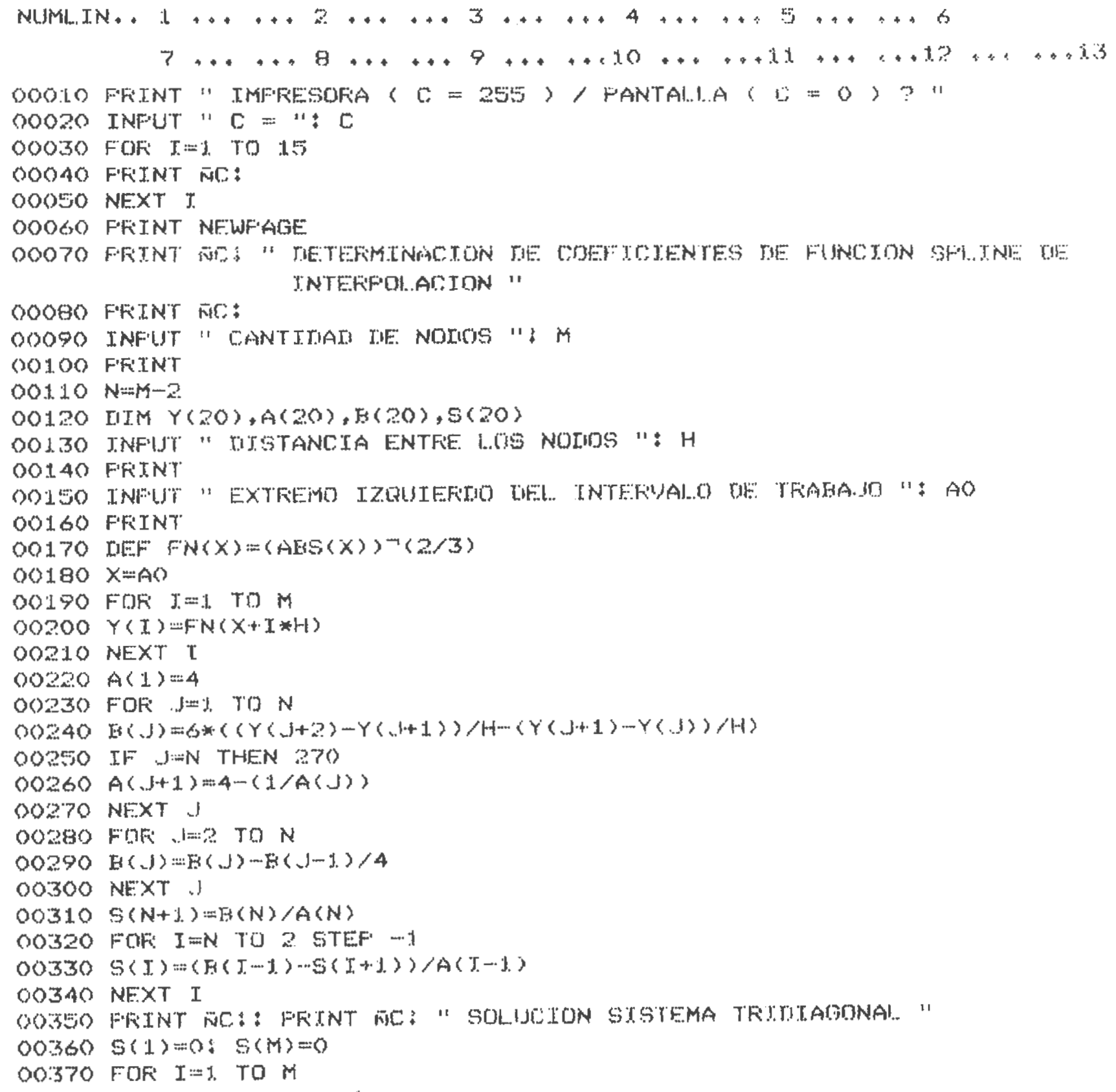




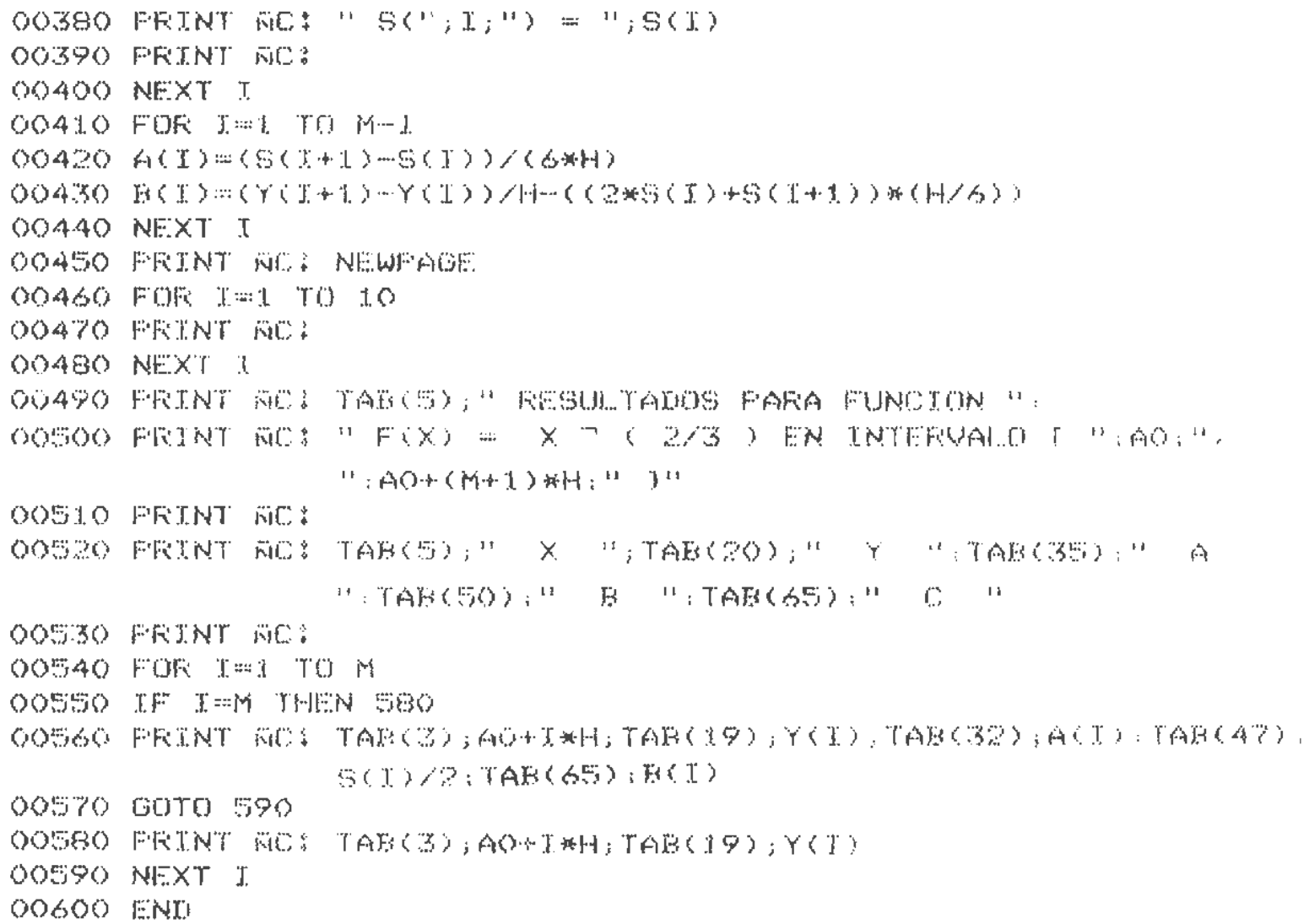




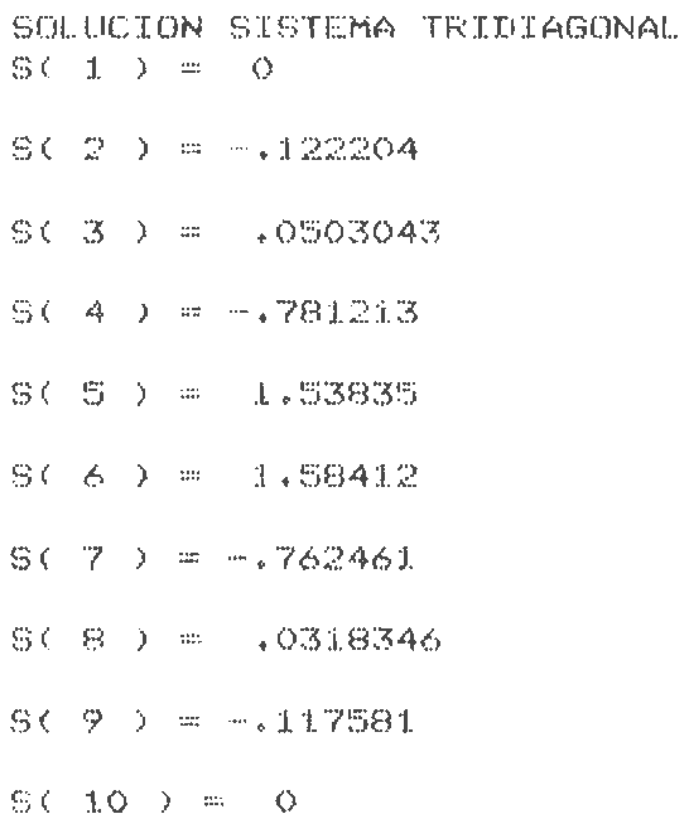




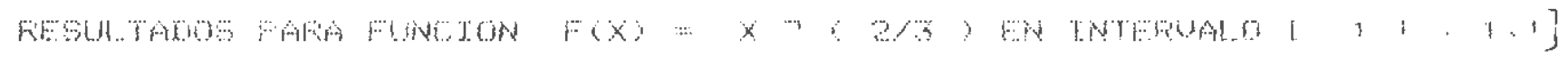
$x$

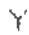

$\rightarrow$

I:

i

\begin{tabular}{|c|c|}
\hline-.9 & $.981 \%$ \\
\hline$\cdots .7$ & .7883724 \\
\hline-45 & $.69 \% \Leftrightarrow 1$ \\
\hline$\cdots, 3$ & .4405141 \\
\hline-.1 & - $21+1444$ \\
\hline .1 & 40.244 \\
\hline .3 & 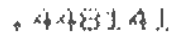 \\
\hline .5 & $.32946 \mathrm{t}$ \\
\hline .7 & $.798,374$ \\
\hline .9 & $.9 \times 2 \times 17$ \\
\hline
\end{tabular}

$\cdots+101937$ $+143 \% 5 \%$

$-.02931$

d. $929 \%$ $.03813 \%$

… +9848

- \$61.913

… - 1.24363

.097984
0

- . 061 tor?

.0251120

... $3900 \%$

.769176

79200?

-. $58123 \mathrm{~L}$

. 0159473

.... $068790 \%$ $\cdots .71490 \%$

. .

- $4544 . \%$

1. $1620 \%$

$.176,56 !$

$1+$ ogx?

- +436

. 7586

- 7 की 


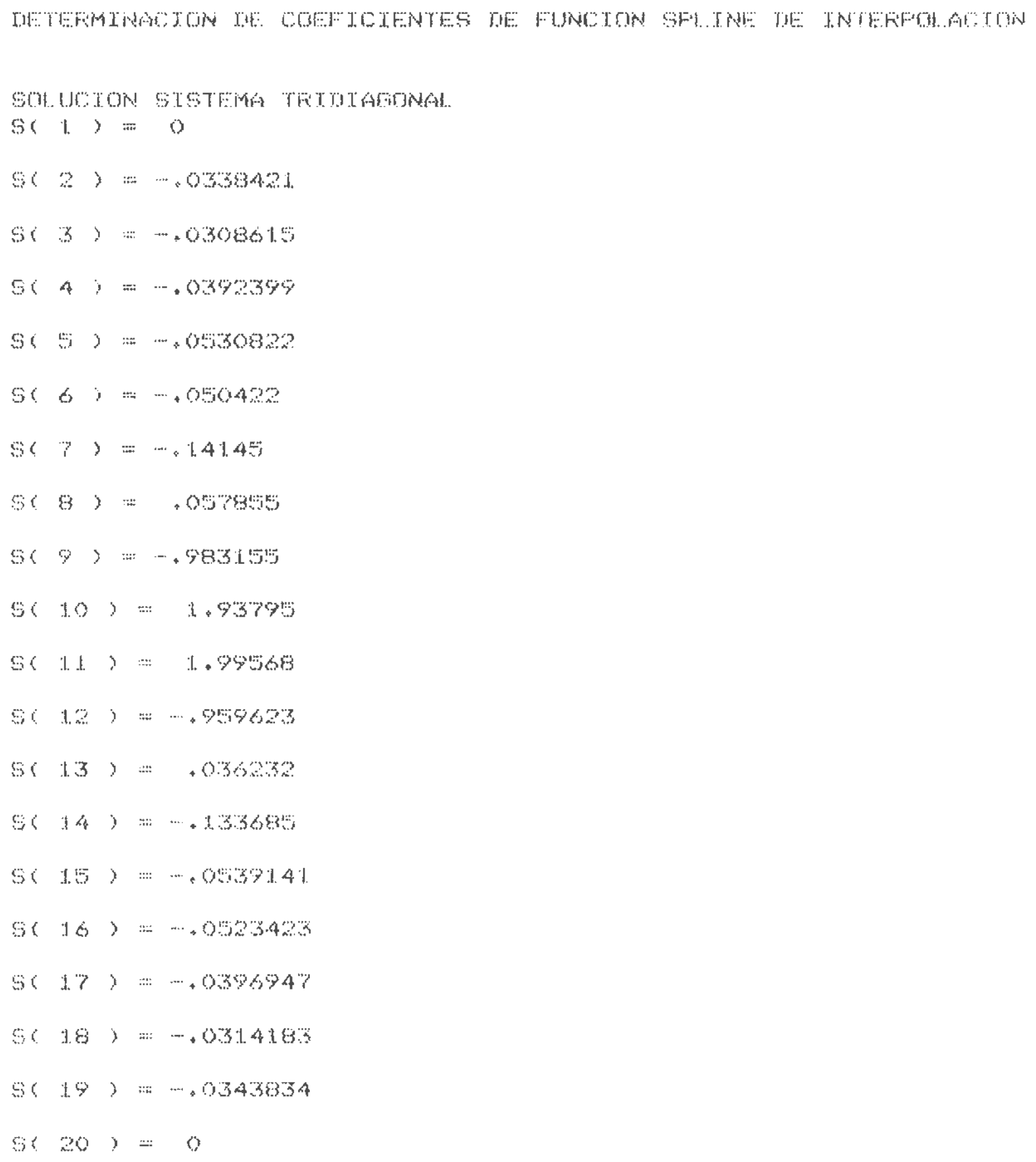




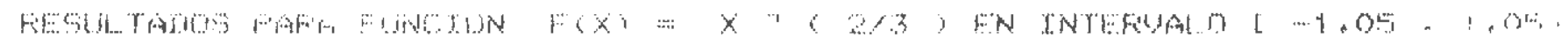

$x$

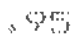
Bi:i

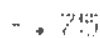
-. 64
- .
, 45
. 30
‥ 2x+y
.
- O
$+15$
$+15$
$+3$
, 35
.43
$+5$
- 65
. 713
- 805
, 4

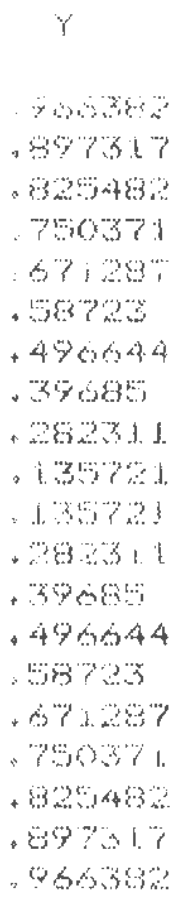

F.

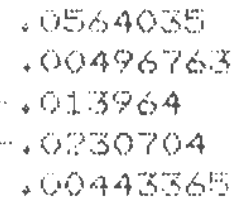

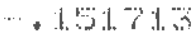

- 5.35174

1.7002

4. 86041

-

$4+8: 26)$

4. 0 क्ष

- . 203195

- 62951.

- 0026496

- $0+10793$

- 0137941

- 00494180

+ 1573056 i)

- . 64 69?

. 51.4030

- $9156 \%$

- क26)

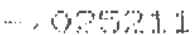

- .670726

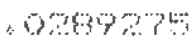

$\cdots=491972$

. $9 \sin \%$

- yopat

... 4

. 019116

- 6069424

$-020 \%$

"0.061\%1

... $01934 \%$

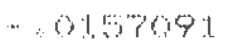

…‥7191. f

$$
\begin{aligned}
& \text { - } \text { कीOFA } \\
& \text { - 716714 } \\
& \text { - } 149479 \\
& \text { - > >3for } \\
& \text {. . } 3.57 \% \\
& \cdots .901,44 \\
& \text {-. } 594197 \\
& -1+1.50 \% 4 \\
& 1+46442 \\
& \text {.. 0978 } \\
& 1+41337 \\
& 1.1704 \\
& \text {. } 959 \% \\
& , 911217 \\
& .94 .3250 \\
& .79 .5235 \\
& \text { : } 729 \% \\
& \text {. } 71.99 ? \\
& .691792
\end{aligned}
$$




\section{BIBLIOGRAFIA.}

[1] UTRERAS, Florencio: "Funciones Spline". Departamento Matemáticas y Ciencias de la Computación. Facultad de Ciencias Físicas y Matemáticas. Universidad de Chile. MA-80-D-225(1980).

[2] LAURENT, P. J.: "Approximation et Optimisation". Ed. Herman. Paris (1972).

[3] CURTIS F., Gerald, WHEATLEY, Patrick: "Applied Numerical Analysis". California Polytechnic State University. Addison-Wesley Publishing Company. 